\title{
Historiadoras británicas del XIX: biografía y edición en las obras de Agnes y Elizabeth Strickland y Mary Anne Everett Green
}

\author{
Nineteenth-century British women historians: Biography and edition in the \\ works by Agnes and Elizabeth Strickland, and Mary Anne Everett Green
}

\author{
Rocío G. Sumillera
}

Universidad de Granada. sumillera@ugr.es

Recibido: 20/03/2017. Aceptado: 14/11/2017

Resumen: El presente artículo analiza la labor de escritura de biografías de mujeres de las prolíficas hermanas Agnes y Elizabeth Strickland, así como la labor de edición llevada a cabo por la reputada historiadora Mary Anne Everett Green tanto en calidad de editora de una selección de epístolas escritas por mujeres y recogidas en los tres volúmenes de Letters of Royal and Illustrious Ladies of Great Britain, como en calidad de editora de 41 volúmenes de los Calendar of State Papers. En el análisis de la obra de Everett Green se discutirán los criterios de edición utilizados en ambos casos, y el proceso por el cual fueron sus criterios de edición de los Calendar los que fijaron el estándar. La labor de estas tres historiadoras se explicará en relación con la de otras autoras de biografías y libros de historia del XIX, poniéndose de manifiesto las preferencias temáticas de sus obras, así como las dificultades editoriales a las que tuvieron que enfrentarse por el hecho de investigar y publicar en una disciplina entonces considerada propia de hombres.

Palabras clave: Calendar of State Papers; Agnes Strickland; Mary Anne Everett Green; biografía; historiadoras victorianas.

\begin{abstract}
The present article discusses the biographies of women of historical significance written by the prolific sisters Agnes and Elizabeth Strickland, and the editorial work developed by the renowned historian Mary Anne Everett Green as editor of the selection of epistles gathered in the three volumes of Letters of Royal and Illustrious Ladies of Great Britain, as well as of 41 volumes of the Calendar of State Papers. Her editorial criteria will be analysed in both cases, and it will be seen how it was she who established and fixed the standard editing criteria in the case of the Calendars. Their work will be contextualised and connected with that of other nineteenth-century women historians, with their thematic preferences when writing history, and with the difficul-

》) Sumillera, Rocío G. 2017. "Historiadoras británicas del XIX: biografía y edición en las obras de Agnes y Elizabeth Strickland y Mary Anne Everett Green”. Quaderns de Filologia: Estudis Literaris 22: 71-88. doi: 10.7203/qfed.22.11253
\end{abstract}


ties they faced when researching and publishing in a discipline at the time considered proper to men.

Keywords: Calendar of State Papers; Agnes Strickland; Mary Anne Everett Green; biography; Victorian historians. 
Here she is-behold her-in the Library of the British museum with her poke Bonnet, her umbrella, her india-rubber overshoes; perhaps sandwiches in the pocket $[\ldots]$ nobody blows a trumpet as she goes home through the dingy streets-instead of her triumphal car, putting up with an omnibus and carrying her notes in a little bag like any ordinary womankind [...] instead of a slow succession of elaborate volumes full of style and pomp, accuracy and importance, it is a shower of pretty books in red and blue, gilded and illustrated, light and dainty and personal, that fall from her hands. In short, it is not Edward Gibbon, but Agnes Strickland, who introduces to our households the reduced pretension of the historic muse (Anónimo, 1855: 437).

En estos términos ridiculizantes y caricaturescos retrataba un anónimo en un número de 1855 de la Blackwood's Edinburgh Magazine a una de las historiadoras más exitosas y populares de la época victoriana: la inglesa Agnes Strickland (1796-1874). Esa "shower of pretty books" a la que el autor se refiere aludiendo tan sólo al color de sus portadas incluye, para el año de 1855, un número desorbitado de publicaciones, entre ellas, los doce volúmenes de The Lives of the Queens of England (1840-1848), que Agnes escribe, como tantos otros títulos, junto con la más oscura figura de su hermana Elizabeth (1794-1875), cuyo nombre no aparece, por deseo personal, en las portadas. Para 1855 también habían visto la luz los dos volúmenes de Victoria: From Birth to Bridal (1840), algunos de los ocho volúmenes de Lives of the Queens of Scotland (1850-1859), y aún quedaban por ser escritos The Bachelor Kings of England (1861), The Lives of the Seven Bishops (1866), Lives of the Tudor Princesses (1868), y Lives of the Last Four Princesses of the House of Stuart (1872), escrita en solitario por Agnes. Esta lista, debe advertirse, no es en absoluto exhaustiva de la producción de ambas, quienes además de escribir ensayos históricos y biografías de mujeres, género por el que fueron más conocidas (o, al menos, por el que Agnes fue conocida), produjeron obras de poesía y de ficción en prosa.

A pesar de todo ello, las críticas que Agnes, la cara visible del dúo, recibía por su obra, toman forma de una crítica, más profunda, por el hecho de ser mujer y escribir historia. Así, en el extracto anterior se

\footnotetext{
* La realización de esta investigación ha sido financiada por el Ministerio de Economía y Competitividad a través del proyecto Las mujeres en la casa de Austria (1526-1567). Corpus Documental (FFI2014-52227-P).
} 
oponen sus obrillas ligeras y superficiales ("light and dainty and personal") a la "slow succession of elaborate volumes full of style and pomp, accuracy and importance" asociada a la producción de historiadores como Edward Gibbon (1737-1794). Agnes parece pues no escribir historia, sino algún tipo de género menor vinculado, de algún modo, con la historia, y en todo caso resultado de "the reduced pretension of the historic muse". Tan habituales y similares eran las críticas en estos términos que bastará mencionar dos más. La primera, publicada en 1851 en Tait's Edinburgh Magazine, afirma que el mérito principal de Lives of the Queens era "the easy and familiar style which reduces the labour of the historical student to a mere pastime", lo cual quedaba lejos de "the scientific accuracy of a professed antiquarian" ([Hosack?] 1851: 238). En otras palabras, la labor de Strickland se presentaba como la de una aficionada que escribía historia a modo de pasatiempo, ajena a la dedicación que se le atribuía al historiador profesional, sesudo y científico. La segunda crítica, el artículo titulado "Miss Strickland's Queen of England", firmado por C. M. S. Phillips, y publicado en 1849 en la Edinburgh Review, acusa a Agnes de mostrar sus preferencias políticas en su obra. Phillips concluye: "Ladies who assume masculine functions must learn to assume masculine gravity and impartiality" (Phillips, 1849: 436). La crítica, pues, no es sólo contra Agnes, sino que va dirigida, por extensión, a las historiadoras en general. Curiosamente, en el prefacio al primer volumen de la serie Lives of the Queens of England, publicado en 1840, las hermanas explícitamente subrayaban la importancia que otorgaban a los hechos frente a las opiniones, y dejaban claro su compromiso por mantenerse fieles al relato de los hechos de la manera más imparcial posible, sin ocultarlos ni alterarlos, sin dejar que las opiniones empañaran la evidencia:

'Facts, not opinions' should be the motto of every candid historian; and it is a sacred duty to assert nothing lightly or without good evidence, of those who can no longer answer for themselves. I have borne in mind the charge which prefaces the juryman's oath-it runs as follows: 'You shall truly and justly try this cause; you shall present no one from malice; you shall excuse no one from favour', etc. (Strickland, 1840: x).

Vuelven a insistir en este punto en el noveno volumen, donde se comprometen a "to say nothing either in the way of praise or censure, which cannot be substantiated by sound evidence" (Strickland, 1846: x). 
Una vez más colocan el énfasis en la importancia de los hechos frente al peso de las opiniones: "Opinions have their date, and change with circumstances, but facts are immutable" (Strickland, 1848: xii).

Las historiadoras del XIX solían elegir temas diferentes a los de sus colegas varones, y entre sus preferencias destacaba, en primer lugar, la biografía de mujeres, seguida de biografías de otros personajes de la realeza y la corte, estudios sobre historia del arte, y traducciones (Maitzen, 1995: 371; Mitchell, 1998: 121)1. Las ya mencionadas The Lives of the Queens of England (1840-1848) y The Lives of the Queens of Scotland (1850-1858), de las hermanas Strickland, así como The Lives of the Princesses of England (1849-1855), de Mary Anne Everett Green, son las más célebres biografías de mujeres del XIX en Inglaterra, aunque la lista continúa y ésta es larga. Antes de ellas, Lucy Aikin había estudiado las cortes de Isabel I, Jacobo I y Carlos I, publicando, entre otros títulos, Memoirs of the Court of Queen Elizabeth (1818) y Memoirs of the Court of King Charles the First (1835). Elizabeth Ogilvy Benger es autora de las memorias de Ana Bolena (1821) y de María Estuardo (1823); Caroline Halsted publica Life of Margaret Beaufort (1839); Katharine Thomson escribe biografías de Sarah, Duchess of Marlborough (1839), y de Charlotte Clayton; Hannah Lawrance escribe Historical Memoirs of the Queens of England $(1838,1840)$ y A History of Woman in England (1843); Martha Walker Freer es autora de la biografía de Maguerite d'Angoulême (1854) y de Jeanne d'Albret (1855); Mrs. Matthew Hall publica Queens before the Conquest (1854), y Elizabeth Cooper, Life and Letters of Lady Arabella Stuart (1866) $)^{2}$. Otras historiadoras no publicaron biografías de mujeres, sino más bien libros sobre historia de las mujeres; así, Julia Kavanagh, por ejemplo, publica Woman in France during the Eighteenth Century (1850) y Women of Christianity (1851). También se comenzaron a compilar entonces diccionarios biográficos de mujeres como el de Matilda Betham, Biographical Dictionary of Celebrated Women of Every Age and Country

\footnotetext{
${ }^{1}$ Ya en el siglo anterior Catharine Sawbridge Macaulay publicó los ocho volúmenes de The History of England from the Accession of James I to that of the Brunswick Line (1763-1783).

${ }^{2}$ Esta lista no es exhaustiva. Para más información sobre historiadoras del XIX no solo británicas sino también norteamericanas y francesas, consúltese (Smith, 1984), y de manera más general (Mermin, 1993).
} 
(1804), o el de Mary Pilkington, Memoirs of Celebrated Female Characters (1804), a la manera de De viris illustribus.

El género de las biografías históricas de mujeres no era solamente el que las historiadoras victorianas cultivaron más, sino el que además se recomendaba como el óptimo para ellas. M. A. Stodart, en un libro titulado Female Writers: Thoughts on Their Proper Sphere, and on Their Powers of Usefulness (1842), afirmaba lo siguiente: "Although history is one of the most useful studies which a woman can pursue, her powers of mind are hardly fitted to enter this field" (Stodart, 1842: 124). No obstante, "the humbler walk of Biography is less unfitted to feminine power" (Stodart, 1842: 128). En otras palabras, el género de la biografía histórica era apropiado para las capacidades de las mujeres por ser menor, y por tanto menos exigente, que la historia tal y como entonces se entendía, a saber, como un relato de los logros de los grandes hombres y de los grandes eventos políticos. Esta idea de la disciplina no solamente excluía a las mujeres, sino también dejaba a un lado otros ámbitos de estudio histórico más allá de la política y más allá de la esfera de lo público, entre otros, la historia cultural y el análisis de la vida cotidiana y la esfera de lo privado ${ }^{3}$.

Las biografías de mujeres no dejaban de suponer un cuestionamiento a la historia tradicional, de la que ellas mismas quedaban excluidas. Estas biografías en cambio mostraban que las mujeres también habían ostentado roles de poder, bien directamente, bien indirectamente, a través de, por ejemplo, la influencia que ejercían sobre los varones de su familia o su círculo social. La biografía que Elizabeth Strickland realiza de María I, en la que cuestiona el mito de reina cruel imperante en las historias protestantes al presentarla más bien como una víctima, es un ejemplo de cómo las biografías escritas por mujeres son en ocasiones contestatarias con respecto al relato histórico tradicional. Y, sin embargo, la escritura de biografías (especialmente de mujeres) se tendía a conceptualizar como una forma de relato de cotilleos y chismes (Maitzen, 1995: 374-375).

Si bien estas biografías por una parte insertaban en el relato de la historia a las mujeres, por otra tenían un fin moralizante, pues muchas

\footnotetext{
${ }^{3}$ Para más información sobre la práctica de la historia por mujeres durante la época victoriana y su relación con la desarrollada por sus colegas varones, consúltese (Crosby, 1991; Maitzen, 1998; Smith, 1998; Laurence, 2000; Levine, 2002).
} 
de las biografiadas se presentaban como modelos a seguir, y en este sentido los relatos reforzaban los ideales de la clase media victoriana acerca de la mujer (Maitzen, 1995: 385-388). Las hermanas Strickland, por ejemplo, ponían de relieve no solo las decisiones políticas de las reinas, sino también sus virtudes. Este carácter ejemplarizante convertía a las biografías en obras especialmente indicadas para un público femenino. El reputado historiador John Lingard (1771-1851), en una carta a su amigo Mr Philip Howard of Corby Castle, comenta así que las obras de Agnes Strickland encontraban a su principal público lector entre las mujeres:

I have snatched a few moments now and then to read Miss Strickland's work, which you had the kindness to send me. It afforded me great pleasure, bringing to my recollection many anecdotes which I had forgotten, and making me acquainted with many that I had never met with at least as far as I can recollect. [...] I should say that Miss Strickland's promises to be a very favourite book, particularly among the ladies, who will be proud of making acquaintance with the female sovereigns of other days (Citado en Strickland, 1887: 47) $)^{4}$.

La historia como disciplina profesional comienza a desarrollarse en Inglaterra en el siglo XIX. Del perfil de las historiadoras de la época se desprenden algunas generalizaciones: muchas eran hijas únicas o no tenían hermanos varones, lo cual explicaba que los recursos económicos de otra forma invertidos en la educación de éstos, fuesen destinados a ellas. Muchas, también, tuvieron padres o algún familiar varón que influyó de manera determinante en sus vidas y en su educación. Por ejemplo, las hermanas Strickland eran parte de una gran familia de seis hermanas y dos hermanos en la que todos, salvo una hermana y un hermano, fueron autores. Todas fueron educadas por su padre, defensor de que niños y niñas recibieran idéntica educación. La muerte de su padre hizo que, por razones económicas, comenzaran a buscarse la vida escribiendo. También en el caso de Green, la segunda de seis hijos de un pastor metodista, el reverendo Robert Wood, es el padre quien se

\footnotetext{
${ }^{4}$ (Strickland, 1887) es la biografía de Agnes, fallecida en 1874, escrita por su hermana pequeña Jane (1800-1888). También esta obra oculta el rol de Elizabeth, que murió en 1875. Existe además una biografía crítica moderna de Agnes Strickland (PopeHennessy, 1940).
} 
encarga de la educación de la hija, cultivando su pasión hacia las letras y la historia, tal como la propia Green recuerda en Memorial of the Rev. Robert Wood, Wesleyan Minister (1854), dedicada a su figura y a su memoria.

Aunque la soltería favorecía el estudio y la investigación, el matrimonio no necesariamente los impedía. Ciertamente, en ocasiones el matrimonio significaba la posibilidad de acceder a bibliotecas y a círculos intelectuales nuevos, la liberación de las restricciones parentales, e incluso un esposo que apoyaba y animaba dicha dedicación, bien por razones intelectuales o movido por necesidades económicas. Ni siquiera, como en el caso de Green, casada con el pintor George Pycock Green, el nacimiento de los hijos supuso el fin de la investigación: la labor de edición de Green se mantiene ininterrumpida durante los años de embarazo y crianza de sus cuatro hijos, la muerte de su hijo en 1876, y la de su marido en 1893 (Mitchell, 1998: 108-112)5. Las historiadoras no obstante se encontraban, a pesar del apoyo familiar que pudieran tener, en clara desventaja con respecto a sus colegas varones: sin posibilidad de una educación universitaria, ni de acceder a muchas sociedades y asociaciones, dependían en mayor medida de una red de contactos más compleja que la estrictamente familiar para consultar archivos y bibliotecas especializadas, y en muchas ocasiones privadas (Mitchell, 1998: 113-115). En el caso de las hermanas Strickland, la importancia que tuvo una sólida red de contactos en su producción como historiadoras es evidente. Lo opuesto de las personalidades de Agnes y Elizabeth hizo que ésta última se mantuviera a la sombra, mientras que Agnes se convertía en la figura pública, en la que firmaba en las portadas de sus obras conjuntas, la que asistía a reuniones y a fiestas, la que se encargaba de cultivar amistades y ganarse invitaciones a eventos y a bibliotecas y colecciones privadas. En efecto, fueron sus amistades y contactos quienes le permitieron acceder a material que de otra manera habría quedado fuera de su alcance. Una vez superadas estas dificultades, las historiadoras se enfrentaban a las aparejadas a la publicación, y a lograr un acuerdo razonable y justo con las casas editoriales, lo cual no siempre sucedía (Mitchell, 1998: 117-118; Tuchman y Fortin, 2014).

\footnotetext{
${ }^{5}$ Mitchell (1998) analiza la vida, la carrera y las obras de una serie de historiadoras nacidas entre 1790 y 1850 , que publicaron entre 1820 y 1880 .
} 
Si las hermanas Strickland son autoras clave en la época victoriana con respecto a la escritura de biografía de mujeres, el caso de Mary Anne Everett Green (1818-1895) supone un reconocimiento a la labor de la historiadora aún mayor, así como unas críticas aún más favorables, y un paso más claro y en firme hacia la profesionalización de la figura de la investigadora en historia. Green destaca en todos los palos que toca: como editora de correspondencia, como autora de biografías de personajes históricos relevantes, $\mathrm{y}$, especialmente, como la más prolífica e influyente editora de los Calendar of State Papers. Ayudada al principio tan sólo por su hermana Esther Wood, cuando a sus colegas se les pagaban asistentes y ayudantes de investigación, Green edita un total de 41 volúmenes de los Calendar.

Seis meses después de su matrimonio, en 1846, y publicados bajo su nombre de soltera (Mary Anne Everett Wood), ven la luz los tres volúmenes de Letters of Royal and Illustrious Ladies of Great Britain: From the Commencement of the Twelfth Century to the Close of the Reign of Queen Mary, su primera obra publicada, a la que volveremos en páginas siguientes. Sin embargo, ya para 1843 Green tenía listo un primer borrador de Lives of the Princesses of England: from the Norman Conquest (1849-1855), una obra de finalmente seis tomos de extensión que cubría desde la Conquista Normanda hasta las vidas de las hijas de Carlos I. La publicación de la misma se pospuso deliberadamente a 1849 por cuestiones puramente editoriales: su editor, Henry Colburn, también lo era de las hermanas Strickland, cuya Lives of the Queens of England terminó de publicarse en 1848. Hasta que el último de los volúmenes de las hermanas no vio la luz, Colburn no comenzó a publicar el primero de los de Green. Para la crítica del momento no cabía duda alguna: la obra de Green era muy superior a la de sus predecesoras. En palabras de Samuel R. Gardiner y J. Bass Mullinger, la de Green era "far more solid and judicious" (Gardiner y Mullinger, 1882: 228). Green consultó documentos de diversa procedencia, custodiados en el Museo Británico, en la biblioteca de Lambeth Palace, en la Tower Records Office, y en colecciones privadas varias, como las de los anticuarios Sir Thomas Phillipps y Dawson Turner. Además, utilizó fuentes procedentes de archivos franceses y colecciones privadas continentales, aprovechando un viaje con su marido al Continente. Los agradecimientos confirman la larga lista de coleccionistas y anticuarios que habían 
accedido a dejarle consultar los fondos de sus bibliotecas, o la habían ayudado con su investigación.

Durante cuarenta años, desde 1855 hasta su muerte en 1895, Green trabajó para la Public Record Office (PRO), produciendo 41 volúmenes de los Calendars of State Papers, Domestic. Su ámbito de estudio comprendía los siglos XVI y XVII: desde el reinado de Eduardo VI, hasta el de Carlos II. Su participación en el proyecto está ligada a Sir Francis Palgrave, Deputy Keeper of the PRO, que la conocía desde la década de los 40, cuando se encontraba inmersa en la preparación de Lives of the Princesses of England. Su conocimiento, destreza en el manejo de documentos, su dedicación, y su dominio de lenguas, le impresionaron profundamente. Cuando se concibió el proyecto de los Calendar y se decidió formar un primer equipo de cuatro editores externos dedicados a compilarlos, Palgrave inmediatamente pensó en ella. Los otros tres editores eran hombres: el anticuario Markham John Thorpe; John Bruce, uno de los miembros fundadores de la Camden Society, y el reverendo John Sherren Brewer, profesor en King's College London. Green era la que menos cobraba de los cuatro, $y$, de todos, la única a la que no le pagaban asistentes y ayudantes de investigación (Krueger, 2003: 74, 77-78).

El fin último de los Calendar era ordenar, clasificar, y organizar cronológicamente los innumerables documentos oficiales de la PRO, entonces desordenados. Luego, además, había que resumirlos. Robert Lemon fue quien bautizó a estos índices-guía con el nombre de calendars, y quien definió los principios editoriales de esta tarea en C.S.P., Dom., Edward I, Mary, Elizabeth, 1547-1580 (1856), el primer volumen de los Calendar que se publica, y que él edita:

The word CALENDAR having been adopted to express the arrangement and description of papers and documents in the order of time; as any other form, without chronological arrangement, would be merely a CATALOGUE (Lemon, 1856: viii).

Prosigue la descripción de su tarea editorial en los siguientes términos:

Each separate paper or document is briefly abstracted, the leading facts stated, and the persons and places to which it relates are mentioned, sufficiently to indicate to what particular subject it belongs. [...] Such 
slight entries rather point out where information may be found, than supply the information itself; but for the fully satisfying the purposes of study recourse must be had to the originals (Lemon, 1856: xi).

El volumen de Green C.S.P., Dom., James I, Volume I, 1603-1610 (1857) fue el primero en aparecer después del inicial de Lemon. El segundo y tercer volumen de Green sobre el reinado de Jacobo I aparecerían al año siguiente, antes de que Thorpe, Bruce o Brewer hubieran completado alguno de los suyos. El estilo de Green, diferente al de Lemon, finalmente se convirtió en el estándar, y por tanto en el seguido por sus continuadores. Para empezar, los resúmenes de Green eran considerablemente más minuciosos y detallados que los escuetos de Lemon, y en ocasiones, incluso, incluían citas de los documentos originales. Los lectores y críticos no dejaron de agradecer esta diferencia (Krueger, 2003: 79-80, 83). La propia Green la advierte en el prefacio al volumen de los Calendar isabelinos, iniciado, aunque jamás terminado, por Lemon, que muere en 1867. Green, encargada de continuar y concluir la tarea de su colega, la explica así:

Mr. Lemon's volumes were compiled on the principle which resulted in the earlier State Paper Calendars, that of indicating rather than describing the contents of the papers. Experience has since proved the advisability of fuller descriptions, such as shall be exhaustive of the important contents of the papers, and shall in most cases preclude the necessity of consulting the originals themselves (Green, 1867: vii-viii).

Además de resumir, Green aportaba información sobre la materialidad del documento original: indicaba el número de páginas que tenía, y el estado en el que se encontraba (si estaba dañado o roto, por ejemplo) ${ }^{6}$. Los primeros cuatro Calendars de Green se publicaron sin

\footnotetext{
${ }^{6}$ Agnes Strickland de hecho se quejaba del lamentable estado en que se encontraban muchos de los documentos originales conservados en archivos públicos: "Every person who has referred to original documents, is aware that it is a work of time and patience to read the MSS. of the Tudor era. Those in the State Paper Office, and in the Cottonian Library, have suffered much from accidents, and from the injuries of time. Water, and even fire, have partially passed over some: in others, the mildew has swept whole sentences from the page $[\ldots]$ It is a national disgrace $[\ldots]$ that so many of the muniments of our history, more especially those connected with the personal expenditure of royalty, should have perished among the ill-treated records of the Exchequer" (Strickland, 1850: x).
} 
introducciones. Sin embargo, con el primer Calendar del reinado de Carlos II, publicado en 1860, sus volúmenes comenzaron a ir precedidos de unas informativas introducciones a la época y a los personajes, algo por lo que Green llevaba años luchando, y a lo que finalmente sus editores accedieron. Esto es, Green es quien concibe (y pelea) por introducciones que contextualicen cada volumen de los Calendar con el fin de ayudar al lector a entender mejor los resúmenes de los documentos. Las introducciones de Green son una mezcla innovadora de historia política y social y de relatos de anécdotas sobre asuntos más mundanos (Krueger, 2006). Juntos, los prefacios de sus volúmenes de los Calendar suman más de 700 páginas. No es casual, pues, que Green sea reconocida como la editora que definió el género de los Calendar como parte de la escritura histórica, creando escuela tanto por sus prefacios, como por su manera de resumir los documentos.

Su labor de edición en su primera obra publicada, los tres volúmenes de Letters of Royal and Illustrious Ladies of Great Britain: From the Commencement of the Twelfth Century to the Close of the Reign of Queen Mary (1846), es también ilustrativa de su rigurosa manera de trabajar con documentos originales que cubren un periodo de cuatro siglos y medio. Tal y como se indica en la portada, las cartas procedían principalmente de originales localizados en "the State Paper Office, the Tower of London, the British Museum, and other State Archives". Cada uno de los volúmenes edita más de 100 cartas con una extensión de más de 300 páginas. Así, el primer volumen, de 372 páginas, reúne 132 cartas; el segundo, de 378 páginas, edita 152 cartas, y el tercero, 158 cartas en 327 páginas, excluyendo índices.

La edición de las cartas presenta siempre la misma estructura: primero aparece el número que Green le asigna a cada carta, luego se especifica quién es el autor y quién el destinatario, y se ofrece la fecha de escritura, el año al menos. El orden de las cartas es cronológico, y en caso de duda acerca de la fecha de alguna, Green indica el que considera más probable, argumentando su datación, y advirtiendo que la fecha es especulativa. Seguidamente, entre corchetes, se indica el archivo en el que se conserva, o bien si la carta ha sido copiada de una publicación anterior en caso de no haber tenido acceso al original. Asimismo, se indica si el texto de la carta es una traducción al inglés de otra lengua. Green es quien realiza las traducciones, que en ningún caso aparecen acompañadas de transcripciones de las cartas en su lengua 
original. Acto seguido, tras tres asteriscos, se ofrece una introducción a la carta de extensión variable. En algunos casos, las introducciones se extienden por varias páginas, son minuciosas y recogen otras cartas escritas (por otras mujeres o por hombres) a propósito de los asuntos que trata la carta editada. En otras ocasiones, las introducciones son más generales y breves, de no más de un párrafo, en el que se contextualiza someramente la carta explicando, por ejemplo, vínculos personales o familiares de las autoras y los destinatarios. Tras una línea horizontal y en un tipo de letra mayor, se transcribe entonces la carta, modernizando la ortografía y la puntuación, o se aporta una traducción al inglés de la misma. Siempre que lo considera preciso, Green añade además notas al pie para información adicional.

Tan sólo el primero de los volúmenes va acompañado de un prefacio en el que Green explica sus decisiones editoriales. En éste, firmado el 20 de noviembre de 1845, Green habla de la dificultad del proceso de selección de las cartas: si con las anteriores al XVI el reto residía en su relativa escasez, con las posteriores el problema era el contrario. Sobre algunas de las primeras Green opina que quizá sean poco interesantes para el lector; su mérito radica en este caso no tanto en su contenido, como en su antigüedad y en su función de mostrar la existencia de una tradición de correspondencia escrita por mujeres: "in forming a continuous series of female epistolary correspondence, their insertion seemed necessary" (Wood, 1846, Vol. I: vi).

Green asegura que su intervención como editora en los textos de las epístolas ha sido deliberadamente mínima: "No liberty whatever has been taken with any of the letters, except in translating those in foreign languages into English, and modernising the ancient orthography" (Wood, 1846, Vol. I: vi). La traducción la realiza de lenguas tan dispares como el latín, el italiano, el español o el francés normando: "it should be observed that the original phraseology has in every instance been retained, and the strictest attention paid to fidelity in the transcripts and to correctness in the translations" (Wood, 1846, Vol. I: vi). Recalca, también, que cualquier intervención suya en el texto es evidente al lector, pues la inserta entre paréntesis:

Not a word has been purposely omitted, inserted, or altered, and, where a supplemental word was necessary to make up the sense, it has been placed within a parenthesis. In those instances where the originals had 
sustained injury from fire, damp, or other casualty, the words supposed to have been effaced have occasionally been conjecturally supplied within brackets (Wood, 1846, Vol. I: vi-vii).

Las fuentes de las cartas son diversas, pues proceden de las bibliotecas de la Torre de Londres y la Office of the Duchy of Lancaster (sobre todo en el caso de los originales más antiguos), y de State Paper Office, el Museo Británico, el College of Arms, la Rolls-House, la Chapter-House, la biblioteca Bodleiana y la Ashmolean Library en Oxford. También algunas proceden de colecciones privadas y de bibliotecas francesas, como la Biliothèque du Roi y los Archives du Royaume, en París.

Green asegura que la mayor parte de las cartas que recoge son inéditas, y que las que han sido publicadas con anterioridad, lo han hecho en obras oscuras y de poca tirada, y por consiguiente difíciles de consultar. Dice además copiar muy pocas de obras ya impresas, esto es, cartas cuyos originales ella no ha visto, lo cual hace como último recurso, por ejemplo porque el original a veces se ha perdido. Este es el caso de las primeras seis cartas que edita: "In these instances the superior interest of the letters induced a departure from the general rule" (Wood, 1846, Vol. I: viii). Otro ejemplo es la epístola escrita por la reina Catalina de Aragón a su confesor, el franciscano John Forest, en 1535, durante el presidio de éste en Newgate (Carta LXXXI, Vol. II). Debido a "the touching piety and resignation of its sentiments" (Wood, 1846, Vol. II: 197), Green afirma que dicha carta merece ser incluida en su edición. Tal y como Green indica en la introducción que precede a la carta, el original de la misma se ha perdido, y es ella quien la traduce al inglés de la traducción anterior al italiano realizada por Girolamo Pollini, quien la publica en su Historia Ecclesiastica della Rivoluzion d'Inghilterra (1594). De Pollini como editor Green habla con respeto, lo que legitima la decisión de su inclusión:

This writer, though a violent Romanist, never tempers with the documents he quotes, as is evident by a careful collation of several of those printed by him with the originals which are still in existence. The following may therefore be considered as the genuine sentiments of the unfortunate Catherine of Aragon, although the language in which they are clothed has unfortunately had to pass through a twofold translation (Wood, 1846, Vol. II: 198). 
La carta de Catalina transmite a su confesor la profunda pena que le produce verle en tal desgraciada situación: "I confess to you that I am consumed by a very great desire to be able to die, either together with you or before you" (Wood, 1846, Vol. II: 199). Concluye con una sentida despedida: "Farewell, my revered father, and on earth and in heaven always have me in remembrance before God. Your very sad and afflicted daughter, Catherine" (Wood, 1846, Vol. II: 200). La respuesta del confesor, Green explica, está recogida en una traducción al inglés publicada en el libro de memorias de Catalina de Aragón escrito por las hermanas Strickland.

Sabemos que Green es autora de todas las traducciones de las cartas que edita, aunque tales traducciones sean luego revisadas por otros. En sus agradecimientos de hecho menciona a "Canon Miguel del Riego, the enthusiastic reviver of the ancient poetry of Spain" (Wood, 1846, Vol. I: xi), quien revisa sus traducciones de varias de las cartas en español de Catalina de Aragón. Quizá no resulte sorprendente que en la larga lista de agradecimientos de Green no aparezca el nombre de una sola mujer; en efecto, Green habla sólo de "distinguished and literary gentlemen who have honoured her with their assistance" (Wood, 1846, Vol. I: ix), bien facilitándole el acceso a bibliotecas privadas, bien mediando para que consiguiera ciertos documentos. Por ejemplo, si al arzobispo de Canterbury le agradece el acceso a Lambeth Library, al Earl of Aberdeen and Viscount Canning le da las gracias por su cortés, sin bien fallido, intento de conseguir del gobierno de España transcripciones de las cartas de la reina Catalina de Aragón localizadas en el Archivo de Simancas.

La selección de cartas de Green no deja de ser curiosa porque en efecto no solamente reúne cartas escritas por princesas, reinas, y nobles, sino que la categoría de "illustrious ladies" le permite además incluir cartas escritas por figuras menos conocidas, y sin embargo en efecto influyentes, como es el caso de mujeres con cargos relevantes en instituciones religiosas. Las firmadas por abadesas y prioras ponen de manifiesto cómo muchas de estas mujeres nombraban y distribuían cargos y puestos, o ejercían su influencia para aupar a otros a conseguirlos, como ilustra la carta de Elizabeth Rede, abadesa de West Malling (Carta LXI, Vol. II). En otros casos, como en el de María, hija de Eduardo I, monja en el convento de Amesbury, las cartas constituyen una manera de pedir favores a sus contactos familiares. Así, hacia 1316, María le escribe a su 
hermano, el rey Eduardo II (Carta XXII, Vol. I), instándole a que le pida a la abadesa de su convento que no nombre a una priora ajena al mismo, esto es, de fuera: "Do this, most dearest brother, that our convent may receive your aid and sustenance in this case as they have always done in their needs" (Wood, 1846, Vol. I: 63). A veces el favor pedido no es para una misma, sino para otros. Green edita, por ejemplo, varias cartas de Catalina de Aragón, que firma como princesa de Gales, enviadas a su padre, Fernando II, en las que busca el beneficio de sus criados. Por ejemplo, en la Carta L (Vol. I), escrita en 1505 y que Green traduce del castellano, Catalina solicita que el hermano del criado que le entrega la epístola a su padre reciba algún puesto en Castilla. Tal favor sería en pago a la gran lealtad del criado, siempre solícito y fiel desde la llegada de Catalina a Inglaterra:

He has served me much since I came into England. He has a brother in Castile; he told me that he is a person in whom there is every merit. I supplicate your highness to send to give him a place in a captainship of men-at-arms, by which your highness will be better served, in which I shall receive most signal favour (Wood, 1846, Vol. I: 128) 7 .

Igualmente, en la carta siguiente (Carta LI, Vol. I), Catalina le pide a su padre que la ayude con las dotes de seis damas que la acompañaron a Inglaterra: ella dice no tener qué darles, y explica que es momento de que se casen.

El prefacio de Green termina apologéticamente, disculpándose por invadir un ámbito, dice, propio de los eruditos del otro sexo. Advierte que no se considera poseedora de ningún talento superior, aunque sí reconoce una gran pasión por el estudio del pasado:

In conclusion, the Editor would beg to offer an apology for the seeming, perhaps real, presumption with which she has ventured upon a field usually occupied only by the learned of the other sex. Inspired by an ardent love for antiquarian literature, and encouraged by the wishes of her friends, she has undertaken her present task, not from any unwarranted conceit of superior talent, but simply because she felt convinced that it was one in which the energy and industry which any one who has time

\footnotetext{
${ }^{7}$ Esta, al igual que otras cartas escritas por Catalina en castellano, se conserva en Egerton MS. 616.
} 
at command may bring to bear upon a given subject were the greatest essentials to success (Wood, 1846, Vol. I: xi-xii).

Green murió en 1895 como vivió, editando sin tregua. A su muerte a los 77 años se encontraba finalizando el proyecto iniciado por su colega John Bruce unos cuarenta años antes, el de edición de los documentos de Carlos I. Fue su sobrina Sophia Crawford Lomas, a quien ella había enseñado a editar, quien completó dicho volumen. Avalado por una biblioteca de ediciones aún hoy imprescindibles para la investigación en historia moderna, su extraordinario talento quedaba entonces fuera de toda duda. Su nombre y sus obras, junto con los de Agnes y Elizabeth Strickland, son imprescindibles en los anales de la investigación en historia en Gran Bretaña, y las vicisitudes de sus trayectorias un recordatorio de las adversidades a las que hubieron de hacer frente por el hecho de ser mujeres dedicadas profesionalmente a un mundo académico y editorial que las consideraba unas intrusas y que en no pocas ocasiones las minusvaloró a pesar de sus éxitos editoriales. Solo una obra formidable ha logrado granjearles el reconocimiento que merecían.

\section{Bibliografía}

[Hosack?, John.] 1851 (April). Miss Strickland's Lives of the Queens of Scotland. Tait's Edinburgh Magazine 18: 238-245.

Anónimo. 1855 (October). Modern Light Literature-History. Blackwood's Edinburgh Magazine 78: 437-451.

Crosby, Christina. 1991. The Ends of History: Victorians and the Woman Question. New York: Routledge.

Gardiner, Samuel R. \& Bass Mullinger, J. 1882. Introduction to the Study of English History. London: Kegan Paul, Trench \& Company.

Green, Mary Anne Everett (ed.). 1867. Calendar of State Papers Domestic: Elizabeth, 1591-94. London: Her Majesty's Stationery Office.

Krueger, Christine L. 2003. Why She Lived at the PRO: Mary Anne Everett Green and the Profession of History. Journal of British Studies 42(1): 65-90.

Krueger, Christine L. 2006. Mary Anne Everett Green and the Calendars of State Papers as a Genre of History Writing. Clio 36: 1-22.

Laurence, Anne. 2000. Women Historians and Documentary Research: Lucy Aikin, Agnes Strickland, Mary Anne Everett Green, and Lucy Toulmin Smith. En Laurence, Anne; Bellamy, Joan \& Perry, Gillian (eds.) Women, Scholarship and Criticism: Gender and Knowledge c. 1790- 
1900. Manchester and New York: Manchester University Press, 125141.

Lemon, Robert. 1856. Calendar of State Papers: Of the Reigns of Edward VI, Mary, Elizabeth (1547-1580). London: Longman, Brown, Green, Longmans \& Roberts.

Levine, Philippa. 2002. The Amateur and the Professional: Antiquarians, Historians, and Archaeologists in Victorian England, 1838-1886. Cambridge: Cambridge University Press.

Maitzen, Rohan Amanda. 1995. "This Feminine Preserve”: Historical Biographies by Victorian Women. Victorian Studies 38: 371-393.

Maitzen, Rohan Amanda. 1998. Gender, Genre, and Victorian Historical Writing. New York and London: Garland Publishing.

Mermin, Dorothy. 1993. Godiva's Rise: Women of Letters in England, 18301880. Bloomington and Indianapolis: Indiana University Press.

Mitchell, Rosemary Ann. 1998. "The Busy Daughters of Clio": Women Writers of History from 1820 to 1880. Women's History Review 7(1): 107-134.

Phillips, C. S. M. 1849. Miss Strickland's Queen of England. Edinburgh Review 89: 435-462.

Pope-Hennessy, Una. 1940. Agnes Strickland: Biographer of the Queens of England. 1796-1874. London: Chatto \& Windus.

Smith, Bonnie G. 1984. The Contribution of Women to Modern Historiography in Great Britain, France and the United States, 1750-1940. American Historical Review 89(3): 709-732.

Smith, Bonnie G. 1998. The Gender of History: Men, Women, and Historical Practice. Cambridge, Mass.: Harvard University Press.

Stodart, M. A. 1842. Female Writers: Thoughts on Their Proper Sphere, and on Their Powers of Usefulness. London: Seeley and Burnside.

Strickland, Agnes. 1841. Lives of the Queens of England, from the Norman Conquest. Vol. I. London: Henry Colburn.

Strickland, Agnes. 1846. Lives of the Queens of England, from the Norman Conquest. Vol. IX. London: Henry Colburn.

Strickland, Agnes. 1848. Lives of the Queens of England, from the Norman Conquest. Vol. XII. London: Henry Colburn.

Strickland, Agnes. 1850. Lives of the Queens of England, from the Norman Conquest. Vol. IV. Philadelphia: Lea and Blanchard.

Strickland, Jane Margaret. 1887. Life of Agnes Strickland. Edinburgh: W. Blackwood.

Tuchman, Gaye \& E. Fortin, Nina. 2014. Edging Women out Victorian Novelists, Publishers, and Social Change. Abingdon: Routledge.

Wood, Mary Anne Everett (ed.). 1846. Letters of Royal, and Illustrious Ladies of Great Britain, from the Commencement of the Twelfth Century to the Close of the Reign of Queen Mary. 3 Vols. London: Henry Colburn. 